\title{
LAS PROFESIONES SANITARIAS EN ZAMORA A MEDIADOS DEL SIGLO XVIII
}

\section{Health Professions in Zamora in the Middle of the Eighteenth Century}

\author{
José Luis HERNÁNDEZ LUIS \\ joluherlu@yahoo.es
}

Fecha de recepción: 23/05/2014

Fecha de aceptación definitiva: 22/07/2014

Para mi padre

RESUMEN: La investigación de la asistencia sanitaria no hospitalaria pasa necesariamente por el análisis de los profesionales de la salud, sus condiciones de trabajo, forma de vida, consideración social y estructura familiar. En este orden de cosas, la atención a mediados del XVIII en los territorios de la actual provincia de Zamora descansaba sobre la figura del cirujano/barbero, que descollaba entre la pluralidad de soluciones asistenciales, aunque esta importancia no se traducía en la pirámide social, coronada desde la perspectiva sanitaria por boticarios y médicos.

Palabras clave: Profesiones sanitarias; Zamora; siglo XVIII.

ABSTRACT: Investigation into non-hospital medical care necessarily has to analyse the work conditions of health professionals, their way of life, social esteem and family structure. In these circumstances, medical care in Zamora during the middle of the $18^{\text {th }}$ century rested on the figure of the barber-surgeon, who stood out among the many possible health care solutions. However, this importance was not reflected in the social pyramid, where, from the health perspective, physicians and chemists were at the top. 
JOSÉ LUIS HERNÁNDEZ LUIS

LAS PROFESIONES SANITARIAS EN ZAMORA A MEDIADOS DEL SIGLO XVIII

Key words: Health professionals; Zamora; $18^{\text {th }}$ century.

\section{INTRODUCCIÓN}

Es, sin duda, la atención sanitaria motivo de inquietud permanente de nuestra sociedad. Nos preocupa la pérdida de la salud y los instrumentos a nuestro alcance para su restablecimiento. Recibimos con alegría, por ello, los avances de la medicina y rechazamos la falta de medios para combatir la enfermedad. Pero, ¿con qué recursos contaban nuestros antepasados para esta misma lucha?

La historiografía española se ha ocupado de esta materia de manera desigual. La investigación de la asistencia sanitaria no hospitalaria en la Edad Moderna ha sido bastante posterior al análisis institucional de los propios hospitales. Su inicio tuvo lugar a mediados de los 70 y sobre todo en los 80, a raíz del despegue de la historia social. Desde entonces han predominado los estudios sobre colectivos sanitarios, en especial médicos y cirujanos, de regiones como Murcia, Extremadura y Aragón ${ }^{1}$.

Para el área castellana a mediados del Siglo de las Luces contamos con el trabajo de Margarita Moretón ${ }^{2}$. Se trata de un estudio estadístico sobre los médicos, boticarios,

1. SÁEz Gómez, José Miguel. Historia de la sanidad municipal en la Murcia de la primera mitad del siglo XVIII. Tesis doctoral, Universidad de Murcia, 1987; Sociología de los profesionales sanitarios murcianos del siglo XVIII. Cartagena: Ayuntamiento de Cartagena, 1992; "Profesionales sanitarios en la Murcia del siglo XVIII: número, evolución y distribución". Asclepio. Revista de Historia de la Medicina y de la Ciencia, 1993, 45, 2, pp. 71-133; ARAGón MATEOs, Santiago. "La atención sanitaria en la Extremadura del siglo XVIII". Alcántara. Revista del Seminario de Estudios Cacereños, 1987, 12, pp. 65-76; López Gómez, José Manuel. "Cirugía y cirujanos en la Mérida del XVIII". Proserpina, 1993, 10, pp. 109-135; Sociología de las profesiones sanitarias en Mérida (1700-1833). Barcelona: Seminari Pere Mata-Promociones y Publicaciones Universitarias, 1994; Granjel, Mercedes. "Médicos y cirujanos en Extremadura a finales del siglo XVIII". Dynamis. Acta Hispanica ad Medicinae Scientiarumque Historiam Illustrandam, 2002, 22, pp. 151-187; “Médicos y élites locales en la sociedad extremeña del siglo XVIII". Llull, 2009, 32, pp. 317-346; FERNÁNDEZ Doctor, Asunción. "Médicos y cirujanos de Zaragoza en la Edad Moderna: su número y estructura sociofamiliar". Dynamis. Acta Hispanica ad Medicinae Scientiarumque Historiam Illustrandam, 1997, 17, pp. 141-164; FERNÁNDEZ DOCTOR, Asunción y ARCARAZO GARCíA, Luis Alfonso. "Asistencia rural en los siglos XVII y XVIII: los tipos de "conducción" de los profesionales sanitarios en Aragón". Dynamis. Acta Hispanica ad Medicinae Scientiarumque Historiam Illustrandam, 2002, 22, pp. 189-208; ARCARAZO GARCíA, Luis Alfonso. La asistencia sanitaria pública en el Aragón rural entre 1673-1750: las conducciones sanitarias de Barbastro. Zaragoza: Institución Fernando el Católico, 2010.

2. Moretón AlOnso, Margarita. Las profesiones sanitarias en Castilla y León (siglo XVIII): análisis sociológico y estadístico. Valladolid: Universidad de Valladolid, 1993. Panorama completado por algunos otros estudios a nivel local: Martínez Flórez, Julio; Calonge García, Francisco y Ballesteros, Montserrat. "La asistencia sanitaria del siglo XVIII en Soria a la luz del Catastro del Marqués de la Ensenada". En Actas de la I Semana de Estudios Históricos de la Diócesis de Osma-Soria. Soria: Diputación Provincial de Soria, 2000, vol. II, pp. 117-128; VICENTE MÉNTRIDA, Marta. Reformas sanitarias y asistenciales en la ciudad de Salamanca durante la segunda mitad del siglo XVIII. Tesis doctoral, Universidad de Salamanca, 2011. 
cirujanos y sangradores en el que, gracias a la información que proporcionan las respuestas generales del Catastro de Ensenada, analiza el número de profesionales, la relación con el censo poblacional y sus emolumentos.

Nuestro propósito con el presente artículo es abordar más en profundidad la asistencia no hospitalaria en una parte concreta de la región, los territorios de la actual provincia de Zamora, durante la etapa previa a la mayoría de las reformas ilustradas. Hasta ahora no disponíamos para ella más que de estudios acerca de hospitales $^{3}$. A tal fin, nos detenemos en el tipo de profesionales de la salud, su patrón de asentamiento, las condiciones de trabajo, la compatibilidad con otras ocupaciones, su patrimonio, el estilo de vida, la consideración que merecían y la estructura de sus familias. Finalmente, el trabajo se completa con una breve alusión a las boticas conventuales, prácticamente desconocidas.

Como fuente se han utilizado las respuestas generales del Catastro de Ensenada que se conservan en el Archivo General de Simancas (en adelante AGS), ya empleadas en estudios de muy diverso tipo ${ }^{4}$. Fundamentalmente hemos manejado la interesante información que contienen las preguntas número 25 (gastos del común), 32 (médicos, cirujanos y boticarios) y 33 (artes "mecánicas") del cuestionario. Esta fuente se ha completado con su complemento lógico, las respuestas particulares que se encuentran en el Archivo Histórico Provincial de Zamora (AHPZa). En ellas hemos localizado buena parte de las declaraciones de médicos y boticarios, entre otros. Para los colectivos muy numerosos, cirujanos y barberos sangradores, nos hemos visto obligados a recurrir a un muestreo atendiendo a criterios de renta y representación geográfica 5 .

Es bien sabido que la investigación con el Catastro de Ensenada presenta problemas, derivados unos de su origen fiscal, que sin duda dio lugar a ocultaciones, y otros del procedimiento de recopilación de datos. Esto último es evidente, por ejemplo, en las respuestas particulares, que recogen lo poseído en cada localidad, con la consiguiente dificultad para reconstruir todo el patrimonio del declarante si se encontraba repartido por varias poblaciones, como era frecuente. Por ello, el historiador ha de extremar las precauciones metodológicas. Aun así, la ingente

3. Véase el estado de la cuestión que expone HernáNDEz LuIs, José Luis. «Panorama hospitalario de Zamora a mediados del siglo XVIII". En prensa.

4. Una propuesta metodológica en OrTiz Gómez, Teresa; Quesada OCHOA, Carmen y ASTRAIN GALLART, Mikel. "El Catastro de Ensenada como fuente para el estudio de las profesiones sanitarias en la España del siglo XVIII". En Actas del XXXIII Congreso Internacional de Historia de la Medicina. Sevilla: Sociedad Española de Historia de la Medicina, 1994, pp. 707-712.

5. La diferenciación entre cirujanos y barberos es complicada en muchas ocasiones, pues incluso las fuentes mencionan indistintamente al mismo individuo de ambas formas. Ni siquiera la legislación de la época arroja luz al respecto, ya que los barberos se ocupaban normalmente tanto de pequeñas cirugías como de rasurar. Cfr. Novísima, libro VIII, título XI, ley VIII. Esto nos ha obligado a detenernos en cada caso para fijar la denominación predominante. 
cantidad de información que aporta lo convierte en imprescindible para el estudio del siglo XVIII en los territorios de la antigua corona de Castilla.

\section{LOS PROFESIONALES DE LA SALUD: COMPOSICIÓN Y ASENTAMIENTO}

En el plano asistencial la Edad Moderna se distingue por el pluralismo de fórmulas. Durante esta época los médicos no monopolizaban el mercado sanitario. Así pues, siempre hubo más cirujanos, barberos, boticarios, comadronas y curanderos que galenos. La elección del ejercitante dependía de la idea que se tuviese sobre su capacidad, la posición en la comunidad y sus honorarios. Era muy frecuente, incluso, el consultar paralelamente a varios profesionales de distinto tipo, con independencia del estatus social del paciente. Además, el papel sanador de la religión siguió existiendo en la Europa moderna, tanto en los países católicos como entre los protestantes ${ }^{6}$.

El estudio de Margarita Moretón, que mencionamos más arriba, muestra una asistencia sanitaria que giraba en tierras zamoranas alrededor de la figura del cirujano o del barbero-sangrador; mal provista de otros profesionales y con una mejor cobertura en los territorios orientales que en los occidentales ${ }^{7}$.

Un análisis más pormenorizado de sus patrones de asentamiento arroja resultados muy interesantes. Zamora era a mediados del siglo XVIII un área agraria con tendencia a la autosuficiencia, salpicada por algunas poblaciones en las que se concentraban las actividades artesanales y mercantiles, muchas veces de subsistencia ${ }^{8}$. Era en estos núcleos urbanos y villas de tipo medio, con funciones de cabecera de comarca o subcomarca, donde radicaban médicos y boticarios (véase Tabla 1). La instalación de galenos en núcleos más pequeños como Fuente el Carnero y Granja de Moreruela debe ponerse en relación con la existencia de dos importantes cenobios: Valparaíso y Moreruela9. En el caso de los albéitares, a la ubicación en núcleos importantes se añadía su residencia en poblaciones vinculadas a ferias ganaderas, vías pecuarias y concentraciones de la cabaña ganadera ${ }^{10}$.

6. Lindemann, Mary. Medicina y sociedad en la Europa moderna, 1500-1800. Madrid: Siglo Veintiuno, 2001, pp. 220-221, 225 y 235-241.

7. Moretón Alonso, Margarita. Las profesiones sanitarias en Castilla y León. Op. cit., pp. 14, 59,61 у 68

8. AlBa López, Juan Carlos y Rueda Fernández, José Carlos. "La industria y el comercio en la Edad Moderna”. En AlBa López, Juan Carlos (ed.). Historia de Zamora. Zamora: Diputación de ZamoraInstituto de Estudios Zamoranos Florián de Ocampo-Caja España, 1995, vol. II, pp. 147-148.

9. AHPZa, Catastro de Ensenada, libro 584, fols. 208r.-208v.

10. Alba López, Juan Carlos y Rueda Fernández, José Carlos. "La industria y el comercio en la Edad Moderna". Op. cit., pp. 160, 167, 179 y 185-187. 
LAS PROFESIONES SANITARIAS EN ZAMORA A MEDIADOS DEL SIGLO XVIII

Tabla 1

ASENTAMIENTO DE LOS PROFESIONALES SANITARIOS

\begin{tabular}{|c|c|c|c|c|}
\hline RESIDENCIA & $\begin{array}{c}\text { N. }{ }^{\circ} \text { DE VECINOS } \\
\text { (LOCALIDAD DE RESIDENCIA) }\end{array}$ & MÉDICOS & BOTICARIOS & ALBÉITARES \\
\hline Alcañices & $148^{*}$ & 1 & 1 & \\
\hline Almeida de Sayago & 246 & 1 & 1 & \\
\hline Benavente & 717 & 2 & 2 & 3 \\
\hline Bóveda de Toro (La) & 135 & & & 1 \\
\hline Cañizal & 239 & & 1 & \\
\hline Carbajales de Alba & 252 & & 1 & \\
\hline Castroverde de Campos & 302 & 1 & 1 & 1 \\
\hline Fermoselle & 663 & 2 & 1 & \\
\hline Fuente el Carnero & 51 & 1 & & \\
\hline Fuentelapeña & 311 & 1 & 1 & 1 \\
\hline Fuentesaúco & 456 & & 1 & 2 \\
\hline Galende & 60 & & & 1 \\
\hline Granja de Moreruela & 62 & 1 & & \\
\hline Lubián & 98 & & & 2 \\
\hline Mombuey & 91 & & & 3 \\
\hline Morales de Toro & 297 & 1 & & 2 \\
\hline Morales del Vino & 265 & & & 1 \\
\hline Palacios de Sanabria & 90 & & & 1 \\
\hline Pinilla de Toro & 212 & & & 1 \\
\hline Pozoantiguo & 146 & & & 1 \\
\hline Puebla de Sanabria & 200 & 1 & 2 & 1 \\
\hline Requejo & 128 & & & 2 \\
\hline S. Miguel del Valle & 170 & 1 & & \\
\hline Toro & 1.491 & 2 & 5 & \\
\hline Vezdemarbán & 440 & & 1 & 2 \\
\hline Villafáfila & 177 & 1 & 1 & \\
\hline Villalobos & 196 & 1 & & 1 \\
\hline Villalpando & $333^{* * *}$ & 1 & 1 & 2 \\
\hline Villamayor de Campos & 215 & & & 1 \\
\hline Villanueva del Campo & $316^{* * *}$ & 1 & & 1 \\
\hline Villardondiego & 117 & & & 1 \\
\hline Zamora & 1.810 & 3 & 3 & \\
\hline Sin identificar & & 1 & & \\
\hline
\end{tabular}

Fuente: AGS, Catastro de Ensenada, Respuestas generales, preguntas 32 y 33 ; *Incluye veinte residentes. ** Las viudas se contabilizan como medio vecino. 
No obstante, era el cirujano o barbero sangrador la figura central de la asistencia sanitaria en la Zamora de la época, sobre todo en el mundo rural. Se trataba, fundamentalmente, de un profesional itinerante (de "acarreo" lo denominan las fuentes), que desde su asentamiento en un pueblo atendía también a otras localidades próximas más pequeñas, además de a caseríos y dehesas ${ }^{11}$.

Existían, empero, otros profesionales de la salud cuya actividad es peor conocida, pero no por ello menos importante. Sin duda relevante era el papel de las matronas. Las matronas, parteras o comadres de los siglos XVII y XVIII eran en toda Europa mujeres expertas en partos y en la salud femenina e infantil. No conformaban un grupo organizado ni poseían una identidad colectiva ${ }^{12}$. En el área que estudiamos algunos concejos importantes como Benavente y Fuentesaúco tenían contratada una matrona. Este último pagaba anualmente a la suya 366 reales. La partera benaventana, en cambio, ganaba al año por su oficio 1.100 reales, aunque suponemos que en esa cantidad van comprendidos también servicios a particulares ${ }^{13}$.

También aparecen registrados enfermeras y enfermeros en villas y ciudades como Benavente, Puebla de Sanabria y Zamora, siempre vinculados a instituciones hospitalarias. Su salario oscilaba entre los 355 y los 730 reales anuales. En este periodo se ocupaban de funciones que a veces les aproximaban al servicio doméstico. No era extraño, incluso, verles atareados en abrir sepulturas en el cementerio del hospital ${ }^{14}$.

Coincidían en estos centros asistenciales con hombres y mujeres que ocupaban un puesto fundamental: el de hospitalero. Los hospitaleros eran retribuidos tanto en metálico como en especie. Se dedicaban a atender a los enfermos, mantener limpias las instalaciones y lavar la ropa ${ }^{15}$.

Mención aparte merecen los saludadores. Enrique Perdiguero ha clasificado los sanadores no oficiales de la Edad Moderna en empíricos (algebristas expertos en fracturas o dislocaciones; hernistas y sacamuelas) y creenciales (ensalmadores que entendían de dolencias externas; saludadores o santiguadores, especialistas

11. Un panorama muy similar al de otras regiones como Extremadura. Véase Granjel, Mercedes. "Médicos y cirujanos en Extremadura a finales del siglo XVIII". Op. cit., pp. 166-174.

12. Desde el siglo XVI eran examinadas por los médicos, bien por decisión colegial o a instancias de los municipios. Acto seguido, se les entregaba una carta de examen que las habilitaba para ejercer en todo el reino. A partir de 1750 serán controladas de manera breve y poco rigurosa por el Protomedicato. Véase OrTIz Gómez, Teresa. "Protomedicato y matronas: una relación al servicio de la cirugía". Dynamis. Acta Hispanica ad Medicinae Scientiarumque Historiam Illustrandam, 1996, 16, pp. 113,115 y 120 .

13. AGS, Catastro de Ensenada, Respuestas generales, libro 654, Benavente, pregunta 25 y libro 358, fols. 95v.-96v.; AHPZa, Catastro de Ensenada, libro 161, fols. 664v.-665r.

14. AHPZa, Catastro de Ensenada, libro 160, fols. 215v.-216v., 230v. y 245r.; libro 1088, fols. 80r.-80v. y libro 1759, fols. 77r.-77v. y 109v.

15. AGS, Catastro de Ensenada, Respuestas generales, libro 626, fol. 59r.; AHPZa, Catastro de Ensenada, libro 19, fol. $54 \mathrm{v}$. 
sobre todo en la rabia; brujas, etc.). Según este mismo autor, la tolerancia de este tipo de prácticas se debía a la carencia de profesionales oficiales y, también, a las creencias de la población sobre la enfermedad ${ }^{16}$. En la provincia de Zamora varios concejos de la comarca toresana y de la Guareña asignaban una cantidad fija a un saludador, que iba de los 20 reales de Villaescusa a los 140 de Villamor de los Escuderos. Parte de su actividad se concentraba en los animales domésticos, como ocurría, por ejemplo, en Cañizal, donde el Común pagaba 60 reales al saludador además de otros 12 por "Saludar" al ganado. En el medio urbano también existían, pues en Toro aparece consignado un saludador que obtenía anualmente unos ingresos de 900 reales $^{17}$.

Aparte de los medios naturales, existían en los siglos modernos otras prácticas sanatorias que eran mezcla de religión y de superstición. Incluso la Iglesia mantuvo clérigos conjuradores que pugnaban por devolver la salud a los endemoniados $^{18}$. En las encuestas zamoranas también aparecen anotadas diversas partidas económicas por conjuros, aunque es posible que también aludan a la costumbre vigente hasta tiempos recientes de ahuyentar o conjurar los temporales con misas y repique de campanas $^{19}$.

\section{CONDICIONES DE TRABAJO}

Muchos de los profesionales sanitarios estaban asalariados por los concejos o por los propios vecinos. En la Edad Moderna el contrato o acto de asalariar que el municipio establecía con un sanitario se denominaba "conducta". Mediante este procedimiento se perseguía conseguir asistencia sanitaria para núcleos pequeños de población sin que el paciente se tuviera que desplazar a una ciudad, considerando, asimismo, que el profesional no se podía sustentar libremente. Si el municipio no disponía de recursos suficientes, se solía imponer un tributo o se sufragaba a través de repartimientos vecinales ${ }^{20}$.

Nos consta que en la provincia de Zamora se redactaban escrituras de conducción, como la convenida entre un médico y el Concejo de la Villa de Fermoselle ${ }^{21}$. Igualmente, el sistema de repartimiento se encontraba vigente en varias

16. Perdiguero Gil, Enrique. "Protomedicato y curanderismo". Dynamis. Acta Hispanica ad Medicinae Scientiarumque Historiam Illustrandam, 1996, 16, pp. 95 y 105-106.

17. AGS, Catastro de Ensenada, Respuestas generales, libro 627, fols. 38v.-39r. y 118v.-119r.; libro 629, fols. 62v.-64r. y libro 626, fol. 60v.

18. SÁnchez Granjel, Luis. Historia politica de la Medicina española. Salamanca: Ediciones Universidad de Salamanca/Real Academia de Medicina de Salamanca, 1985, pp. 208-209.

19. AGS, Catastro de Ensenada, Respuestas generales, libro 627, fols. 143v.-144r. y libro 363, fol. 262 r.

20. FERnÁNDEZ DOCTOR, Asunción y ARCARAZO GARCía, Luis Alfonso. "Asistencia rural en los siglos XVII y XVIII". Op. cit., pp. 190-191.

21. AHPZa, Catastro de Ensenada, libro 490, declaración 493. 
localidades zamoranas, tanto para médicos como para cirujanos y barberos ${ }^{22}$. El profesional concertado con el concejo adquiría así la obligación de atender a todos los vecinos o solo a los pobres de solemnidad ${ }^{23}$.

En otras ocasiones, el profesional estaba ajustado con los vecinos. Fue esta una práctica que se aplicó a lo largo de toda Europa ${ }^{24}$. En tales casos cada vecino solía entregar al ejercitante cierta cantidad de grano. A veces la cantidad se reducía a la mitad si el residente era una viuda ${ }^{25}$.

También el ejercitante podía convenirse con una institución eclesiástica concreta, bajo la obligación de atender a sus miembros ${ }^{26}$. Finalmente, el profesional podía estar concertado al mismo tiempo con el concejo y con alguna institución religiosa, sin renunciar tampoco al libre ejercicio ${ }^{27}$.

El examen de las cantidades devengadas que aparecen detalladas en las fuentes nos muestra, en primer lugar, que el asalariar a un sanitario, al igual que ocurría en otras regiones ${ }^{28}$, era una práctica predominantemente rural, ya que en las poblaciones importantes, por disponer de más profesionales, no fue tan necesario (véase Tabla 2).

22. AGS, Catastro de Ensenada, Respuestas generales, libro 630, fols. 52v.-53r. y libro 627, fols. 75v.-76r.

23. AGS, Catastro de Ensenada, Respuestas generales, libro 657, San Miguel del Valle, pregunta 32 y libro 627, fols. 118v.-119r.

24. Lindemann, Mary. Medicina y sociedad en la Europa moderna. Ob. cit., p. 191.

25. AGS, Catastro de Ensenada, Respuestas generales, libro 666, Faramontanos de Tábara y libro, 664, Santa Clara de Avedillo, en ambos pregunta 32.

26. AGS, Catastro de Ensenada, Respuestas generales, libro 669, Villanueva de Campeán, pregunta 32 .

27. AGS, Catastro de Ensenada, Respuestas generales, libro 357, fol. 277r. Una situación muy similar a la que se daba, por ejemplo, en la provincia de Palencia, donde la mayoría de los cirujanos estaban asalariados por los concejos, por los vecinos o de ambas maneras, sin excluir el ejercicio libre. Véase Moretón Alonso, Margarita. "Los cirujanos palentinos de la segunda mitad del siglo XVIII". En Calleja González, María Valentina (ed.). Actas del II Congreso de Historia de Palencia. Palencia: Diputación Provincial de Palencia, 1990, vol. IV, pp. 739-740.

28. ARCARAzo García, Luis Alfonso. La asistencia sanitaria pública en el Aragón rural entre 1673-1750. Op. cit., p. 426. 
JOSÉ LUIS HERNÁNDEZ LUIS

LAS PROFESIONES SANITARIAS EN ZAMORA A MEDIADOS DEL SIGLO XVIII

Tabla 2

CANTIDADES DEVENGADAS POR CONCEJOS O VECINOS (EN REALES)

\begin{tabular}{|c|c|c|c|c|}
\hline Localidad & Médico & Barbero-cirujano & Saludador & Matrona \\
\hline Alcañices & 440 & & & \\
\hline Barcial del Barco & & 200 & & \\
\hline Bóveda de Toro (La) & & & 44 & \\
\hline Cañizal & & 216 & 72 & \\
\hline Castroverde de Campos & 2.600 & & & \\
\hline Cernadilla & & 227 & & \\
\hline Domez & & 176 & & \\
\hline Donado & & 120 & & \\
\hline Faramontanos de Tábara & & 104 & & \\
\hline Fermoselle & 2.315 & $200 / 1.800$ & & \\
\hline Formariz & & 90 & & \\
\hline Fuentelapeña & 2.500 & & & \\
\hline Fuentesaúco & & & 120 & 366 \\
\hline Gramedo & & 161 & & \\
\hline Letrillas & & 110 & & \\
\hline Monumenta & & 210 & & \\
\hline Moreruela de Tábara & & 492 & & \\
\hline Palazuelo de Sayago & & 240 & & \\
\hline Pinilla de Fermoselle & & 150 & & \\
\hline Porto & & 200 & & \\
\hline Rionegro del Puente & & 150 & & \\
\hline Sagallos & & 150 & & \\
\hline S. Cristóbal de Entreviñas & & 792 & & \\
\hline S. Miguel del Valle & 1.972 & 968 & & \\
\hline S. Pedro de la Nave & & 640 & & \\
\hline S. Román de los Infantes & & 96 & & \\
\hline Sta. Clara de Avedillo & & 1.207 & & \\
\hline Sta. Eulalia de Río Negro & & 275 & & \\
\hline Tábara & & 792 & & \\
\hline Toro & 5.000 & & & \\
\hline Torre del Valle & & 300 & & \\
\hline Val de Sta. María & & 144 & & \\
\hline Valdemerilla & & 88 & & \\
\hline Valdescorriel & 603 & & & \\
\hline Valparaíso & & 165 & & \\
\hline Vegalatrave & & 220 & & \\
\hline Villabrázaro & & 450 & & \\
\hline
\end{tabular}


JOSÉ LUIS HERNÁNDEZ LUIS

LAS PROFESIONES SANITARIAS EN ZAMORA A MEDIADOS DEL SIGLO XVIII

\begin{tabular}{|l|r|r|r|r|}
\hline Localidad & Médico & Barbero-cirujano & Saludador & Matrona \\
\hline Villaescusa & & 200 & 20 & \\
\hline Villalonso & & 1.344 & & \\
\hline Villamayor de Campos & & 2.400 & & \\
\hline Villamor de la Ladre & & 40 & & \\
\hline Villamor de los Escuderos & & 120 & 140 & \\
\hline Villanueva de Valrrojo & & 225 & & \\
\hline Villar de Farfón & & 82 & & \\
\hline Villárdiga & & 264 & & \\
\hline Vivinera & & 70 & & \\
\hline Zafara & & 90 & & \\
\hline Media & 2.204 & 374 & 79 & 366 \\
\hline
\end{tabular}

Fuente: AGS, Catastro de Ensenada, Respuestas generales, preguntas 25 y 32 .

Es posible observar, asimismo, el alto coste de los médicos frente al de los sangradores y los cirujanos. Ello explica por qué los servicios de los primeros, únicos profesionales con formación universitaria en la España de mediados del siglo XVIII, solo estuviera al alcance de algunas poblaciones y que los segundos fuesen los profesionales más frecuentemente concertados. En el último escalón retributivo se encontraban empíricos como los saludadores ${ }^{29}$.

Aunque se dispusiera de un profesional convenido con el concejo, un grupo de vecinos podía llegar a un acuerdo con otro. Cuando había varios profesionales del mismo tipo, los pacientes acudían al que juzgaban con mayor habilidad ${ }^{30}$.

Las profesiones sanitarias mayoritarias, salvo los médicos, que eran los únicos con titulación universitaria, se organizaban a la manera gremial en aprendices, oficiales (a menudo denominados "mancebos") y maestros. Los aprendices, muchachos jóvenes, a veces no percibían remuneración alguna, pues probablemente se entendían suficientemente recompensados con el aprendizaje del oficio. Los mancebos, en cambio, solían ser retribuidos también con su manutención aparte del salario ${ }^{31}$. La parquedad de las fuentes al respecto solo nos permite inferir que oficiales y aprendices estaban mejor pagados en el medio urbano que en el medio rural, en consonancia, igualmente, con los ingresos de los maestros.

29. No obstante, el salario medio de los profesionales conductados que ejercieron en el ámbito zamorano fue muy inferior al registrado, por ejemplo, entre sus colegas extremeños. Véase GRANJEL, Mercedes. "Médicos y cirujanos en Extremadura a finales del siglo XVIII". Ob. cit., pp. 179-180 y 183-186.

30. AGS, Catastro de Ensenada, Respuestas generales, libro 667, Monfarracinos y Perilla de Castro, en ambos pregunta 32.

31. AGS, Catastro de Ensenada, Respuestas generales, libro 630, fol. 53r.; libro 358, fol. 100v. y libro 655, Puebla de Sanabria, pregunta 33; AHPZa, Catastro de Ensenada, rollo 1211, fols. 111v.-112r. y libro 28 , fol. 891 r. 
Entre los profesionales sanitarios descubrimos relaciones de parentesco que nos informan acerca de la transmisión del oficio dentro de la familia. Así encontramos hijos que ayudaban a los padres, padres e hijos desarrollando idéntica función en el mismo centro hospitalario, como es el caso de una enfermera del Hospital de la Piedad, y hermanos compartiendo ocupación ${ }^{32}$.

Escasos son, por último, los datos que contienen las fuentes en cuanto a otras condiciones de trabajo. Sabemos, por ejemplo, que los albéitares que ejercían también el oficio de herradores laboraban entre 180 y 300 días al año ${ }^{33}$.

\section{RENTA Y PATRIMONIO}

Margarita Moretón ha puesto de relieve como los médicos eran los profesionales mejor retribuidos, seguidos en este orden por los boticarios, los cirujanos y los barberos sangradores ${ }^{34}$.

Generalmente, en Zamora estaba peor remunerado el ejercicio libre que la práctica sanitaria bajo acuerdo o "conducta". También los ingresos por el ejercicio de la medicina en sus diversas variantes dependían de la habilidad o prestigio adquiridos por el profesional ${ }^{35}$. Por la práctica de su oficio los sanitarios eran retribuidos, ora en metálico, ora en especie, sobre todo en grano, pero también con lana, vino o algún animal doméstico, y a veces con el pago del arrendamiento de la vivienda que ocupaban ${ }^{36}$.

Con relativa frecuencia los profesionales compatibilizaban sus funciones sanitarias con otras actividades. La principal, sin duda, la agricultura, pero igualmente aparecen otras ocupaciones. Hallamos algunos barberos ejerciendo como fieles de fechos en algunos núcleos rurales. Las tareas sanitarias tampoco eran obstáculo para ocupar puestos judiciales, como era el caso del médico de Granja de Moreruela, asimismo alcalde mayor de la localidad. Finalmente, varios profesionales se dedicaban al comercio de lienzos, aceite, especias u ofrecían posada, sin que la pertenencia de algunos al estado noble fuese impedimento ${ }^{37}$.

32. AGS, Catastro de Ensenada, Respuestas generales, libro 71, fols. 261v.-262r. y libro 654, Villardeciervos, pregunta 32; AHPZa, Catastro de Ensenada, libro 161, fol. 660v.

33. AGS, Catastro de Ensenada, Respuestas generales, libro 71, fols. 261v.-262r. y libro 655, Galende, pregunta 33.

34. Para las retribuciones de estos colectivos remitimos a MORETón AlONSO, Margarita. "Las profesiones sanitarias en Castilla y León". Op., cit., p. 15.

35. AGS, Catastro de Ensenada, Respuestas generales, libro 666, Fermoselle, pregunta 32; libro 71, fols. 256r.-256v. y libro 358, fol. 100v.

36. AGS, Catastro de Ensenada, Respuestas generales, libro 627, fols. 535r.-535v. y libro 664, Venialbo, pregunta 32; AHPZa, Catastro de Ensenada, libro 584, fols. 208r.-208v.

37. AHPZa, Catastro de Ensenada, libro 571, fols. 305r.-312v.; libro 642, fol. 94v.; libro 843, fols. 410v.-411r.; libro 1763, fols. 52r.-52v. y libro 1088, fols. 361v.-362r.; AGS, Catastro de Ensenada, Respuestas generales, libro 665, Cubillos, pregunta 33. También en Extremadura médicos y barberos desarrollaron actividades no sanitarias en el terreno agrario, comercial y de la Administración. Véase 
Sus actividades contribuyeron a que formaran un patrimonio, muy diferente en función del grupo sociosanitario. Vamos a aproximarnos a él brevemente según lo permiten las respuestas particulares, ya que no recogen el conjunto de bienes poseídos, sino los radicados en una localidad concreta.

En este sentido, los boticarios aparecen como el grupo poseedor del patrimonio más rico y diverso: abundante ganado ovino, colmenas, tierras de secano, árboles frutales, viviendas y construcciones auxiliares (bodegas, pajares, paneras). Incluso consta algún apotecario ocupado en tareas crediticias. Los cirujanos y barberos muestran, por lo común, una hacienda mucho más modesta: alguna cabeza de ganado ovino o vacuno, algún inmueble y unas pocas tierras de secano o viñas. Sorprende, finalmente, el pobre patrimonio declarado por los médicos, que nos muestra una mayor dependencia del ejercicio profesional y, quizá, una mayor movilidad, con menos arraigo en las poblaciones de residencia ${ }^{38}$.

Sobre estas propiedades acumuladas pagaban cuotas de foros, tanto a cofradías y capellanías como a miembros de la baja nobleza; aniversarios y derechos señoriales. Algunos de sus bienes se encontraban, además, hipotecados en censos, contraídos también con instituciones eclesiásticas ${ }^{39}$.

Los sanitarios que alcanzaban una avanzada edad para la época y no podían ejercer su oficio dependían a menudo económicamente de sus hijos. Así ocurría, por ejemplo, con un cirujano de Carbajales, de setenta años de edad, que era mantenido por un hijo sacerdote que tenía en Zamora ${ }^{40}$.

\section{ESTILO DE VIDA}

A fin de aproximarnos a la forma de vida de los profesionales sanitarios tomaremos como muestra su vivienda: la localización, el régimen de tenencia y la valoración, en la localidad más importante de la provincia, la ciudad de Zamora (véase Tabla 3).

López Gómez, José Manuel. "Cirugía y cirujanos en la Mérida del XVIII". Op. cit., pp. 119-120; Sociología de las profesiones sanitarias en Mérida. Op. cit., pp. 54-55 y 60-62.

38. AHPZa, Catastro de Ensenada, libro 52, fols. 295v.-296r.; libro 1764, fols. 31r.-32v.; libro D 22, fols. 13r.-13v.; libro 161, fols. 355v.-356r. y libro 1762, fols. 851r.-851v. José Manuel López Gómez y Mercedes Granjel han demostrado como los médicos extremeños del siglo XVIII no podían contar con patrimonios considerables si se limitaban al ejercicio profesional. Cuando existía riqueza estaba fundada en patrimonios preexistentes y rendimientos extraprofesionales. Véase López GómEz, José Manuel. Sociología de las profesiones sanitarias en Mérida. Op. cit., pp. 60-62 y 69; GrAnJEL, Mercedes. "Médicos y élites locales en la sociedad extremeña del siglo XVIII". Op. cit., p. 328.

39. AHPZa, Catastro de Ensenada, libro 1764, fol. 30r.; libro 843, fol. 412r.; libro 571, fols. 313r.313v. y libro 241, fol. 423r.

40. AHPZa, Catastro de Ensenada, libro 24, fol. 426v. 
LAS PROFESIONES SANITARIAS EN ZAMORA A MEDIADOS DEL SIGLO XVIII

Tabla 3

ASENTAMIENTO DE LOS PROFESIONALES SANITARIOS EN LA CIUDAD DE ZAMORA

\begin{tabular}{|l|l|c|}
\hline \multicolumn{1}{|c|}{ UBICACIÓN DE LA VIVIENDA } & RÉGIMEN DE TENENCIA & VALORACIÓN (REALES) \\
\hline Médicos & & 400 \\
\hline C/ del Riego & Arrendamiento & 400 \\
\hline Rúa de los Francos & Arrendamiento & 242 \\
\hline Sin identificar & Arrendamiento & \\
\hline Boticarios & & 110 \\
\hline C/ de la Reina & Foro & 400 \\
\hline C/ de la Renova & Foro & 300 \\
\hline C/ del Puente & Foro & 550 \\
\hline Pza. Mayor & Foro & \\
\hline Cirujanos & & 132 \\
\hline C/ de la Cárcava & Foro & 200 \\
\hline Barberos & & 44 \\
\hline C/ de Balborraz & Arrendamiento & 165 \\
\hline C/ de Carniceros & Arrendamiento & 99 y 166 \\
\hline C/ de la Cárcava & Arrendamiento & 99 y 300 \\
\hline C/ de la Feria (x2) & Arrendamiento & 165 \\
\hline C/ de la Plata (x2) & Arrendamiento & 99 \\
\hline C/ de la Renova & Arrendamiento & 99 \\
\hline C/ de Quebrantahuesos & Arrendamiento & 137 y 187 \\
\hline Colación de Sta. Lucía (x2) & Arrendamiento & 165 \\
\hline Plazuela de S. Juan de Puertanueva (x2) & Arrendamiento & No consta \\
\hline Pza. de Sta. Lucía & Arrendamiento & 132 \\
\hline Puebla de la Feria & No consta & \\
\hline Rúa de los Francos & Foro & \\
\hline Sin identificar & Arrendamiento & \\
\hline
\end{tabular}

Fuente: AHPZa, Catastro de Ensenada, libros 1762-1764.

Comenzando por el asentamiento de los sanitarios, podemos observar su preferencia por la zona de mayor importancia artesanal y comercial de la ciudad. Todos se agrupan en torno a lo que en el siglo XVIII era el eje económico de la ciudad, que comenzaba en la puerta de la Feria, subía por la calle de la Cárcava (actual Costanilla), ocupaba la plaza Mayor y aledaños, para bajar por la calle de Balborraz hacia la plaza de Santa Lucía y el puente Mayor ${ }^{41}$.

41. Alba López, Juan Carlos y Rueda Fernández, José Carlos. "La industria y el comercio en la Edad Moderna". Op. cit., p. 189. La concentración en áreas céntricas y comerciales era común en la época, como se ha demostrado para Barbastro (Huesca). Véase ArCARAzo GarCía, Luis Alfonso. La asistencia sanitaria pública en el Aragón rural entre 1673-1750. Op. cit., pp. 190 y 219-222. 
En lo concerniente al régimen de tenencia, e íntimamente relacionado con la localización, es evidente el predominio del arrendamiento, mayoritario siempre en las zonas más importantes, desde el punto de vista económico, de las ciudades. Destaca, sin embargo, la inclinación de los boticarios por habitar casas en régimen de foro, tal vez buscando una mayor estabilidad para la ubicación de sus negocios. El valor de las viviendas, por último, nos indica el superior nivel de vida de los boticarios y de los médicos con respecto a los barberos y los cirujanos.

El análisis de la estructura y de las dimensiones de la vivienda pone de manifiesto diferencias entre los distintos grupos socioprofesionales, así en el medio rural como en los núcleos urbanos. Los boticarios, por ejemplo, poseían las mejores casas. Sus viviendas contaban con uno o varios patios, numerosas piezas, desván, pajar, bodega, corral, pozo, caballeriza y huerto, donde en algún caso se cultivaban rosales, romeros y otras plantas para elaborar medicamentos. Las casas de los médicos, por el contrario, eran algo más pequeñas. Solían componerse de varias piezas altas y bajas, pozo, pajar, corral y caballeriza. De más modestas dimensiones eran, finalmente, las casas de los barberos y de los cirujanos, conformadas por varias piezas y corral. Eran casi siempre de planta baja, aunque en los núcleos urbanos podían presentar sus estancias repartidas en varias alturas ${ }^{42}$.

Con cierta frecuencia aparecen animales compartiendo estos espacios con sus dueños, especialmente caballerías, de raza equina o asnal, para el transporte de los miembros de la familia, y cerdos, camperos o de ceba, para complemento de la dieta ${ }^{43}$.

El servicio doméstico es, por último, otro excelente indicador del nivel de vida de los sanitarios ${ }^{44}$. Como vemos en la tabla 4, también los boticarios estaban a la cabeza en este aspecto, seguidos por los médicos. La media de sirvientes de los cirujanos y de los barberos demuestran, al contrario, un estilo de vida más modesto.

Tabla 4

EDAD Y ESTRUCTURA FAMILIAR (MEDIAS)

\begin{tabular}{|l|r|r|r|}
\hline \multicolumn{1}{|c|}{ GRUPO } & \multicolumn{1}{c|}{ EDAD } & \multicolumn{1}{c|}{ HIJOS } & \multicolumn{1}{c|}{ SERVICIO DOMÉSTICO } \\
\hline Médicos & 43 & 1,46 & 1,38 \\
\hline Boticarios & 45 & 2,33 & 1,73 \\
\hline Cirujanos & 44 & 0,90 & 1 \\
\hline Barberos & 40 & 1,41 & 0,41 \\
\hline
\end{tabular}

Fuentes: AHPZa, Catastro de Ensenada, Respuestas particulares.

42. AHPZa, Catastro de Ensenada, libro 241, fol. 422r.; libro 1764, fol. 31r.; libro D 22, fols. 341v. y 722 r.; libro 52, fol. 286r.; libro 24, fol. 426 r. y libro 1763 , fol. 54 r.

43. AHPZa, Catastro de Ensenada, libro 1628, fol. 377r. y libro 1762, fol. 854 r.

44. Granjel, Mercedes. "Médicos y élites locales en la sociedad extremeña del siglo XVIII". Op. cit., p. 331. 


\section{CONSIDERACIÓN SOCIAL}

Aunque la mayoría de los profesionales sanitarios pertenecían al estado llano, también había entre ellos un puñado de hidalgos, especialmente entre los boticarios. Alguno, incluso, ejerciendo además otras tareas mercantiles. En otro caso aparece claramente el origen castrense de la pertenencia al estamento privilegiado, como le sucedía a un cirujano que disfrutaba del fuero militar de inválido ${ }^{45}$.

Con independencia de su adscripción estamental, las fuentes muestran la distinta estima social que tenían los diferentes grupos profesionales. Así ocurre, por ejemplo, con las borrosas categorías de barberos y de cirujanos, integrados en alguna ocasión los primeros en el grupo de los jornaleros y los segundos en el de labradores (agricultores propietarios) ${ }^{46}$.

En este sentido, el tratamiento de don, descartado por la historiografía más reciente como signo indudable de pertenencia en el siglo XVIII a los estamentos privilegiados, es, sin embargo, prueba válida en esta época de la consideración que merecía el individuo en una determinada comunidad. Así pues, todos los médicos que ejercían en la provincia de Zamora y solo algunos boticarios constan con dicho tratamiento. Nunca los otros profesionales. Obviamente, la estima en este caso derivaba de su preparación, ya que los médicos en ese momento eran los únicos sanitarios con formación universitaria ${ }^{47}$.

La naturaleza de las fuentes apenas nos permite rastrear las relaciones extraprofesionales entre los distintos grupos de sanitarios, que con toda probabilidad se daban. Como botón de muestra, aparecen varios ejemplos de profesionales viviendo en foro o arrendamiento en casas pertenecientes a otros sanitarios o a instituciones de carácter asistencial ${ }^{48}$.

\section{ESTRUCTURA FAMILIAR}

La media de edad de los sanitarios zamoranos era elevada para la época, 43 años, y sin grandes distancias entre los diferentes grupos socioprofesionales, pues los más jóvenes, los barberos, tenían una media de 40 años, y los mayores, los

45. AHPZa, Catastro de Ensenada, libro 1088, fol. 361v. y rollo 1211, fol. 109v.; AGS, Catastro de Ensenada, Respuestas generales, libro 665, Casaseca de las Chanas, pregunta 33.

46. AGS, Catastro de Ensenada, Respuestas generales, libro 668, San Miguel de la Ribera, pregunta 33 .

47. Este reconocimiento fue utilizado por algunos médicos en el siglo XVIII para establecer relaciones con la élite e integrarse en ella. Véase GRANJEL, Mercedes. "Médicos y redes sociales: mecanismos de poder de la profesión médica en el siglo XVIII". Asclepio. Revista de Historia de la Medicina y de la Ciencia, 2012, LXIV, 2, p. 460. Por lo que respecta a los boticarios, su prestigio irá aumentando a lo largo del barroco. En 1650, por ejemplo, la botica dejó de ser un oficio mecánico para convertirse en un arte. Véase MuÑoz CAlvo, Sagrario. Historia de la Farmacia en la España moderna y contemporánea. Madrid: Síntesis, 1994, pp. 178-179.

48. AHPZa, Catastro de Ensenada, libro 1763, fol. 54r. y libro 161, fol. 359v. 
boticarios, 45 (véase tabla 4). Esta situación probablemente se deba al tiempo transcurrido hasta el establecimiento por su cuenta, después de pasar por la categoría de aprendices y mancebos u oficiales. Quizá en el caso de los médicos haya que pensar en el asentamiento previo en otros destinos con carácter formativo.

Entre los profesionales sanitarios predominaba el estado civil de casado. Del espectro con el que hemos trabajado solamente alrededor del 10\% aparecen como solteros y una cifra similar (11\%) había enviudado.

Mayoritariamente sus familias eran de tipo nuclear, formadas por los dos cónyuges y sus vástagos, aunque en algún caso se acompañan de otros familiares como sobrinos y cuñados. El número de hijos era pequeño, en torno a uno o dos de media, destacando como más fértiles los boticarios (véase Tabla 4). Una vez más, comprobamos que el número de hijos guardaba asimismo relación con el nivel de vida, al igual que otros aspectos ${ }^{49}$.

\section{LAS BOTICAS CONVENTUALES}

Tenemos constancia de al menos tres boticas en instituciones religiosas de la ciudad de Zamora a mediados del siglo XVIII: en los Conventos de San Jerónimo y de Santo Domingo y en el Colegio de San Andrés, de los jesuitas. Fuera de la ciudad existían boticas en los Monasterios de Moreruela y de Valparaíso ${ }^{50}$. Aunque es muy probable que funcionara alguna más, especialmente en las comunidades conventuales de Toro, que no aparecen en los documentos examinados.

Tales establecimientos eran administrados por los propios religiosos, auxiliados por mancebos ${ }^{51}$. Como era habitual en la época, las boticas servían tanto a las comunidades como a los habitantes de sus núcleos de asentamiento y de los lugares próximos. Por ejemplo, el boticario del Monasterio de Valparaíso estaba ajustado con los vecinos de Mayalde por tres cargas de trigo ${ }^{52}$. Los servicios farmacéuticos al entorno social inmediato les proporcionaban unos apreciables ingresos anuales (véase Tabla 5), que eran mucho mayores, como se puede comprobar, en el medio urbano que en el rural.

49. En este sentido, la comparación con los profesionales zaragozanos nos hace patente familias más cortas y, con ello, un menor nivel de vida. Véase FERnÁndez Doctor, Asunción. "Médicos y cirujanos de Zaragoza en la Edad Moderna". Op. cit., pp. 158-161.

50. AGS, Catastro de Ensenada, Respuestas generales, libro 663, Zamora, pregunta 32; libro 654, Granja de Moreruela, preguntas 32 y 33 y libro 667, Mayalde, pregunta 32.

51. AGS, Catastro de Ensenada, Respuestas generales, libro 654, Granja de Moreruela, pregunta 32; AHPZa, Catastro de Ensenada, libro 1758, fol. 611v.

52. PESSA DE Oliveira, Enrique. "Para o estudo da saúde conventual no início do século XIX: as boticas". Asclepio. Revista de Historia de la Medicina y de la Ciencia, 2011, LXIII, 1, pp. 138-140. AGS, Catastro de Ensenada, Respuestas generales, libro 667, Mayalde, pregunta 32. 
Tabla 5

INGRESOS DE LAS BOTICAS CONVENTUALES (EN REALES)

\begin{tabular}{|l|l|r|}
\hline \multicolumn{1}{|c|}{ LOCALIDAD } & \multicolumn{1}{c|}{ INSTITUCIÓN } & \multicolumn{2}{c|}{$\begin{array}{c}\text { INGRESOS ANUALES } \\
\text { DE LA BOTICA }\end{array}$} \\
\hline Granja de Moreruela & Monasterio de Sta. María & 2.000 \\
\hline Zamora & Colegio de S. Andrés & 6.095 \\
\hline Zamora & Convento de S. Jerónimo & 5.547 \\
\hline Zamora & Convento de Sto. Domingo & 4.547 \\
\hline
\end{tabular}

Fuente: AHPZa, Catastro de Ensenada, libro 641, fol. 14r.; libro 1758, fols. 603 v., 611v. y 625r.-625v.

La competencia de las boticas conventuales suscitaba la protesta de los apotecarios seglares. Comprensible si se comparan los ingresos que acabamos de ver con los 2.860 reales, por ejemplo, que le reportaba la botica a D. Alonso Rodríguez, farmacéutico de Zamora, que se queja de la competencia en su declaración ${ }^{53}$.

\section{CONCLUSIONES}

La asistencia no hospitalaria en la Zamora de mediados del siglo XVIII, como en otras zonas, se distinguía por el pluralismo de profesionales dedicados a tareas sanitarias ${ }^{54}$. Destacaba, empero, por encima de todos el cirujano/barbero, profesional itinerante, que estaba concertado en la mayoría de los casos con los concejos o con los vecinos. Su papel era fundamental en el mundo rural, ya que médicos y boticarios se agrupaban en los núcleos urbanos y en las villas de tipo medio. En este sentido, la situación asistencial de las localidades más importantes era también mejor por concentrar, además, los hospitales de curación ${ }^{55}$. En estas tareas sanitarias la presencia de la mujer se limitaba a las funciones de

53. AHPZa, Catastro de Ensenada, libro 1764, fol. 32v. Tal intrusismo llegaba a suscitar prolongados pleitos, como el sostenido en Barcelona por los apotecarios contra el Convento de Santa Catalina Mártir a caballo de los siglos XVI y XVII. Véase JorDi GONZÁLEZ, Ramón. Aportació a la història de la Farmacia catalana (1285-1997). Barcelona: Fundació Uriach 1838, 1997, pp. 59-62.

54. El pluralismo médico en Cataluña, por ejemplo, ha sido tratado por ZARZoso, Alfons. "El pluralismo médico a través de la correspondencia privada en la Cataluña del siglo XVIII". Dynamis: Acta Hispanica ad Medicinae Scientiarumque Historiam Illustrandam, 2001, 21, pp. 409-433. En sus páginas 412 y 418-420 hallaremos un estado de la cuestión sobre el desarrollo de este concepto por la historiografía clásica anglosajona: Margaret Pelling, Charles Webster, David Gentilcore, Laurence Brockliss, Colin Jones y Roy Porter, amén de otros.

55. HeRnández LuIS, José Luis. "Panorama hospitalario de Zamora a mediados del siglo XVIII". En prensa. 
matrona, hospitalera y enfermera, las dos últimas categorías vinculadas siempre a instituciones hospitalarias ${ }^{56}$.

Respecto al nivel de vida de los profesionales de la salud, cuestiones como el patrimonio, la vivienda o el tamaño de la familia nos muestran a los boticarios como el grupo con el mejor pasar, seguidos por los médicos y terminando por los cirujanos y los barberos. Aun así, los sanitarios zamoranos muestran siempre un menor estatus que sus homólogos extremeños y aragoneses.

En cuanto a la consideración social, los médicos eran los profesionales más estimados, probablemente por ser los únicos sanitarios con preparación universitaria. Por otra parte, como hemos apreciado, la pertenencia de algunos profesionales sanitarios a los estamentos privilegiados no era obstáculo para el ejercicio de actividades mercantiles.

En resumen, si nos atenemos a las retribuciones y a la consideración social, la pirámide socio-sanitaria zamorana estaría constituida en orden descendente por los médicos, los boticarios, los cirujanos y los barberos. Panorama muy similar, sin embargo, al dibujado para otras zonas de Castilla ${ }^{57}$. Si nos ceñimos, en cambio, al nivel de vida, los boticarios sustituirían claramente a los galenos en la cúspide de la pirámide social sanitaria.

\section{BIBLIOGRAFÍA}

Alba López, Juan Carlos y Rueda Fernández, José Carlos. "La industria y el comercio en la Edad Moderna". En Alba López, Juan Carlos (ed.). Historia de Zamora. Zamora: Diputación de Zamora-Instituto de Estudios Zamoranos Florián de Ocampo-Caja España, 1995, vol. II, pp. 147-215.

ARAGÓn MATEOS, Santiago. "La atención sanitaria en la Extremadura del siglo XVIII". Alcántara. Revista del Seminario de Estudios Cacereños, 1987, 12, pp. 65-76.

ARCARAZO GARCía, Luis Alfonso. La asistencia sanitaria pública en el Aragón rural entre 1673-1750: las conducciones sanitarias de Barbastro. Zaragoza: Institución Fernando el Católico, 2010.

CABRÉ I PAIRET, Montserrat y ORTIZ Gómez, Teresa (eds.). Sanadoras, matronas y médicas en Europa, siglos XII-XX. Barcelona: Icaria, 2001.

FERnÁNDEZ DOCTOR, Asunción. "Médicos y cirujanos de Zaragoza en la Edad Moderna: su número y estructura sociofamiliar. Dynamis. Acta Hispanica ad Medicinae Scientiarumque Historiam Illustrandam, 1997, 17, pp. 141-164.

FERnÁNDEZ DOCTOR, Asunción y ARCARAZO GARCía, Luis Alfonso. "Asistencia rural en los siglos XVII y XVIII: los tipos de "conducción" de los profesionales sanitarios en

56. Para el papel femenino en las profesiones sanitarias recomendamos el conjunto de estudios coordinado por CABRÉ I PAIRET, Montserrat y OrTIZ Gómez, Teresa (eds.). Sanadoras, matronas y médicas en Europa, siglos XII-XX. Barcelona: Icaria, 2001.

57. Martínez Flórez, Julio; Calonge García, Francisco y Ballesteros, Montserrat. "La asistencia sanitaria del siglo XVIII en Soria a la luz del Catastro del Marqués de la Ensenada". Op. cit., p. 124. 
Aragón". Dynamis. Acta Hispanica ad Medicinae Scientiarumque Historiam Illustrandam, 2002, 22, pp. 189-208.

Granjel, Mercedes. "Médicos y cirujanos en Extremadura a finales del siglo XVIII". Dynamis. Acta Hispanica ad Medicinae Scientiarumque Historiam Illustrandam, 2002, 22, pp. 151-187.

Granjel, Mercedes. "Médicos y élites locales en la sociedad extremeña del siglo XVIII". Llull, 2009, 32, pp. 317-346.

GRANJEL, Mercedes. «Médicos y redes sociales: mecanismos de poder de la profesión médica en el siglo XVIII". Asclepio. Revista de Historia de la Medicina y de la Ciencia, 2012, LXIV, 2, pp. 435-466.

HERNÁNDEZ LUIS, José Luis. "Panorama hospitalario de Zamora a mediados del siglo XVIII". En prensa.

JoRdi GONZÁlez, Ramón. Aportació a la bistòria de la Farmacia catalana (1285-1997). Barcelona: Fundació Uriach 1838, 1997.

Lindemann, Mary. Medicina y sociedad en la Europa moderna, 1500-1800. Madrid: Siglo Veintiuno, 2001.

LóPez Gómez, José Manuel. "Cirugía y cirujanos en la Mérida del XVIII". Proserpina, 1993, 10, pp. 109-135.

LóPEz Gómez, José Manuel. Sociología de las profesiones sanitarias en Mérida (1700-1833). Barcelona: Seminari Pere Mata-Promociones y Publicaciones Universitarias, 1994.

Martínez Flórez, Julio; Calonge García, Francisco y Ballesteros, Montserrat. "La asistencia sanitaria del siglo XVIII en Soria a la luz del Catastro del Marqués de la Ensenada". En Actas de la I Semana de Estudios Históricos de la Diócesis de Osma-Soria. Soria: Diputación Provincial de Soria, 2000, vol. II, pp. 117-128.

MORETÓn ALONSO, Margarita. "Los cirujanos palentinos de la segunda mitad del siglo XVIII". En CAlleja González, María Valentina (ed.). Actas del II Congreso de Historia de Palencia. Palencia: Diputación Provincial de Palencia, 1990, vol. IV, pp. 737-745.

MORETón ALONSO, Margarita. Las profesiones sanitarias en Castilla y León (siglo XVIII): análisis sociológico y estadístico. Valladolid: Universidad de Valladolid, 1993.

MuÑoz Calvo, Sagrario. Historia de la Farmacia en la España moderna y contemporánea. Madrid: Síntesis, 1994.

Ortiz Gómez, Teresa; Quesada Ochoa, Carmen y Astrain Gallart, Mikel. "El Catastro de Ensenada como fuente para el estudio de las profesiones sanitarias en la España del siglo XVIII". En Actas del XXXIII Congreso Internacional de Historia de la Medicina. Sevilla: Sociedad Española de Historia de la Medicina, 1994, pp. 707-720.

ORTIZ GÓMEZ, Teresa. "Protomedicato y matronas: una relación al servicio de la cirugía". Dynamis. Acta Hispanica ad Medicinae Scientiarumque Historiam Illustrandam, 1996, 16, pp. 109-120.

Perdiguero Gil, Enrique. "Protomedicato y curanderismo". Dynamis. Acta Hispanica ad Medicinae Scientiarumque Historiam Illustrandam, 1996, 16, pp. 91-108.

Pessa De Oliveira, Enrique. "Para o estudo da saúde conventual no início do século XIX: as boticas". Asclepio. Revista de Historia de la Medicina y de la Ciencia, 2011, LXIII, 1, pp. 123-154.

SÁez Gómez, José Miguel. Historia de la sanidad municipal en la Murcia de la primera mitad del siglo XVIII. Tesis doctoral, Universidad de Murcia, 1987.

Sáez Gómez, José Miguel. Sociología de los profesionales sanitarios murcianos del siglo XVIII. Cartagena: Ayuntamiento de Cartagena, 1992. 
SÁEz Gómez, José Miguel. "Profesionales sanitarios en la Murcia del siglo XVIII: número, evolución y distribución". Asclepio. Revista de Historia de la Medicina y de la Ciencia, 1993, 45, 2, pp. 71-133.

SÁnChez Granuel, Luis M. ${ }^{a}$. Historia politica de la medicina española. Salamanca: Ediciones Universidad de Salamanca/Real Academia de Medicina de Salamanca, 1985.

VICENTE MÉNTRIDA, Marta. Reformas sanitarias y asistenciales en la ciudad de Salamanca durante la segunda mitad del siglo XVIII. Tesis doctoral, Universidad de Salamanca, 2011.

ZARZOSO, Alfons. "El pluralismo médico a través de la correspondencia privada en la Cataluña del siglo XVIII". Dynamis: Acta Hispanica ad Medicinae Scientiarumque Historiam Illustrandam, 2001, 21, pp. 409-433. 